\title{
An Investigation of Analytic Decision During Driving Test
}

\author{
Samir Ghouali \\ Fac of Technology, STIC Lab, \\ University of Tlemcen, \\ Algeria
}

\author{
Yassine Zakarya Ghouali \\ Fac of Economics, Business and \\ Management Sciences, POLDEVA \\ Lab, University of Tlemcen, \\ Algeria
}

\author{
Mohammed Feham \\ Fac of Technology, STIC Lab, \\ University of Tlemcen, \\ Algeria
}

\begin{abstract}
To examine the long-term causality between Cardiorespiratory Electromyography Galvanic signals for 17 drivers taken from Stress Recognition in Automobile Drivers database.

Methods: Two statistical methods, co-integration to reveal an eventual existence of a long-term relationship between ECG (Electrocardiograph), EMG (electromyography), GSR (galvanic skin resistance), heart rate (HR) and respiration, well as the Application of the model of Granger causality.

Results: ECG shows certain dependence to EMG, GSR, heart rate and respiration. The results for ECG dependent suggest that an increase of $1 \%$ in EMG, FOOTGSR, HAND GSR, HR and RESPIRATION implies a variation of ECG which take a value respectively of $0.016248 \%, 0.007241 \%, 0.028366 \%, 0.000511 \%$ and $0.000110 \%$ in the within dimension based on the FMOLS (Fully Modified Ordinary Least Squares). With same way, the result for ECG suggest that an increase of $1 \%$ in EMG, FOOT GSR, HAND GSR, HR and RESPIRATION implies a variation of ECG which take a value respectively, of $0.065684 \%$, $0.014534 \%, 0.032800 \%, 0.000304 \%, 0.005986 \%$ in the between dimension based on the same method. The results of panel Granger causality show a bi-directional relationship between ECG and FOOT GSR, HAND GSR and respiration signals, it must be noted as a unidirectional causality from EMG to ECG.

Conclusion: This study shows the long-term interaction between the bio signals, and reveal how the understanding of these interactions can help the doctors to understand the risks that may exist between these interactions. The main advantage of a multidimensional and multivariate model is to solve a multitude of problems that prevent doctors to treat the patients better and is not the case for studies in two dimensions.
\end{abstract}

Keywords-Panel Co-integration; Panel Granger Causality; FMOLS and DOLS Estimators; Cardiorespiratory electromyography galvanic signals

\section{INTRODUCTION}

Actually, ECG signal is not independent of the other physiological signals, and medical explanations can attest to this. Thus, this concept of interactions between the physiological signals must be well formulated and analyzed. In this context, this article was devoted to the presentation of a strategy to analyze the interactions between biomedical signals in order to develop an approach to diagnosis.

The choice of the mathematical models, resulting from the physiological signals, for the characterization of such or such pathology becomes crucial. In order to widen the detection of these anomalies, we proposed a statistical analysis of the physiological signals to research factors of causality between them.

Traffic has become a major human activity; its development has resulted in huge infrastructure projects of communication used for a variety of uses, implementing very different vehicles. The industry associated with them has played and continues to play a significant economic role.

The evidence is that the main causes of accidents were related to a lack of driver vigilance. This lack of vigilance is, in fact, the result of many factors that are identified as inattention, drowsiness, and errors related to fatigue. Medication, drugs, alcohol, health accidents are all causes of accidents deserve to be treated specifically because they have become the main source of road accidents [1], (Algeria was ranked fourth in the Arab countries, in terms of road traffic accidents, with a heavy balance sheet that was estimated at 44,907 accidents resulted in the death of 4540 people and left 69,582 injured nationally [2]).

Many teams, for nearly twenty years, have been mobilized to better understand the origins of this drowsiness and to detect the occurrence as early as possible in order to take the necessary security provisions: braking, stopping.

In the automotive world, this goal is called "active safety" and complements the "passive safety" which aims to reduce the extent of damage in case of an accident.

The situation today is that many approaches have been explored, leading to important scientific and technological developments on-board sensors and diagnostic methods. However, we can say that these developments are not yet arrived at an operational stage, mainly because of diagnostic errors: false alarms, fault detection ... that remain present and hinder implementation confident developed devices. The analysis of this situation shows three emergencies:

- Continue the validation work required by multiplying the test

- Develop methods and tools for detecting

- Perfect the valuation procedures for accurate comparison of results with embedded diagnostics "reference" systems expertise from physiological signals. 
It is on this last point that took our work on "Contributions of mathematical models to analyze short and long-term objective physiological signals, the vigilance of car drivers." It enriches existing analysis of biological signals in three ways:

- The accuracy of features extraction of the real signal and it is not our purpose in this work.

- Develop telemedicine applications to monitoring the health status of the patient

- process automation implemented in order to provide medical experts the possibility of multiple testing and diagnostics "referring" to monitor the couple "drivervehicle," and anticipate the occurrence of a hazard sufficiently well characterized to trigger an alarm or automatically make a rescue maneuver [3].

The panel data models knew these twenty last years a very sharp enthusiasm. This passion resulted in a true explosion amongst academic work founded on the panel date models. The aspects of the transposition of time series problems to the panels are detailed in what follows.

Also named the structure with double dimensions, the study in panel brings information richer as that available in time series [35]. Indeed, it constitutes a particularly invaluable statistical source for the analysis of the dynamic behaviors.

The profit which results from all that, is the possibility of modelling more complex individual behaviors and dynamic alternatives. The distinction between the dynamic macro/micro effects Interactional, is done by the addition of a temporal dimension, It is one of the advantages to the recourse to the data of panel as Hsiao [36] indicates it. It is necessary to discuss two significant factors such as the decomposition of the total variability of the data and the increase amongst degrees of freedom which aims to decrease the collinearity between the data.

The panel date has a major asset concerning the number of data. To use a significant number of data increases the degree of freedom and decreases the collinearity between the variables.

The panel data thus provide the possibility of deducing the individual behavior while making use of the behaviors of the other individuals. The use of data of panel also makes it possible to reduce the frequent problems in time series of collinearities between the explanatory variables thanks to the possibility of introducing inter individual differences. These individual effects have the second advantage of being able to identify and take account of the unobservable effects.

Obviously, nothing is perfect and each model contains limits. Among the disadvantages of the panel of data, we can cite an incomplete panel, a panel says not rolled, problems of heteroscedasticity and/or autocorrelation of the random variations, but it is not always easy to correct or avoid these disadvantages.

For this configuration, we are leaning models on panel data, where we considered a double dimension: an individual dimension and a temporal dimension. Individual dimension represents the patient and temporal dimension represents the studied physiological signals.

The taking into account of biomedical data also generated an increase in the temporal dimension which results in a transposition of the questions usually asked into time series, such as the stationnarity, the non-linearity or the temporal stability of the relations.

In this context, the use of data panel models at the same time makes possible to combine the advantages of working on the panel data and solving the problems of nonlinearity, heterogeneity and temporal instability. More precisely, these models authorize the existence of dynamic individual distinct being able to evolve in time while taking account of asymmetries.

These changes are therefore an interesting solution to meet the new challenges posed by the use of panel data. However, this field is relatively recent what implies that certain current debates of time series as non-stationariness according to nonlinearity is not posed yet in panel data.

The issue of using these changes for forecasting purposes is also addressed in this article.

This article initially proposed an outline of methods devoted to the principal tests of unit roots, of Co-integration on panel data, models of estimates as well as the use of causality within the meaning of Granger in panel. This research experienced a great development since work pioneers of Levin and Lin and is today the object of multiple applications at the empirical level. The theoretical framework, which is the base of any empirical study, brings contents of legitimacy to our problems, as it is used to clarify the concepts and makes it possible to define each concept.

We worked out the analysis of the physiological signals containing a mathematical model as a panel, pertaining to the same family of Granger. This model exploits Co-integration on data panel and allows the quantification short and long terms if it exists using estimators FM-OLS and DOLS. Within this framework, we brought an analytical study of the interactions between the physiological signals of the drivers of vehicles using a modeling of the interactions between the cardiorespiratory signals galvanic electromyographies.

The goal of these studies is to propose the direction of causality between the physiological signals as well as the quantification of the rates of causality if it exists in Panel. We carried out the analysis of each scenario according to the following stages:

- To check if there exists a long-term relation between the vital signals.

- To quantify the rate of convergence of this long-term relation.

- To define the direction of causality between the signals on the basis of causality of Granger as a Panel.

- To understand the impact of the signals on the longterm heart. 
Numerous studies have been devoted to the evaluation of causality; several applications are omnipresent in areas ranging from the economy $[4,5]$, directed information theory in networks [6], brain imaging field [7], genetics [8] and especially the analysis of biological systems, with a very special emphasis on the neural field $[9,10]$, the study of cardiac signals $[11,12,13,33,34]$ and cardiorespiratory interactions $[14,15]$.

In this article, we will look at studied panel data causality and panel Co-integration of a number of physiological signals, derived from the Stress Recognition in Automobile Drivers dataset [16] from the PhysioBank database [17], ,this approach was then applied to electrocardiogram (ECG), electromyography (EMG), galvanic skin resistance (GSR) measured on the hand and foot, heart rate (HR) and RESPIRATION.

This paper is organized as follows: In Section 2, we will establish the data used and the methodology. In section 3, we present the approach of Co-integration, in section 4, 5 and 6 the estimated long-term relationship, and Granger causality tests respectively. Finally, we lead an analysis, scientific discussion, conclusion and a projection of perspectives.

\section{VARIABLES, DATA ANALYSIS AND METHODOLOGY}

\section{A. Variables}

Electrocardiogram (ECG) is a recording of the electrical activity of the heart. The initial diagnosis of heart attack is usually made through observation of a combination of clinical symptoms and characteristic ECG changes [26].

Electromyography (EMG) is a diagnostic procedure to assess the health of muscles and the nerve cells that control them (motor neurons).

The Galvanic Skin Response (GSR) is defined as a change in the electrical properties of the skin. The measurement is relatively simple, and has a good repeatability. Therefore, the GSR measurement can be considered a simple and useful tool for examination of the autonomous nervous system function [28].

Heart rate (HR) is the speed of the heartbeat measured by the number of poundings of the heart per unit of time typically beats per minute (bpm).

Respiration is the biochemical process in which the cells of an organism obtain energy by combining oxygen and glucose, resulting in the release of carbon dioxide.

\section{B. Data Analysis}

There is a set of multi-parameter data instances from healthy volunteers in The Stress Recognition in Automobile Drivers dataset [16] from the PhysioBank database [17], these data were taken while the volunteers were driving on a designed path including highways and city streets in the region of Boston. The aim of this work is to find out the feasibility of automated recognition of stress on the basis of the recorded signals, which include electrocardiogram (ECG), electromyography (EMG), galvanic skin resistance (GSR) measured on the hand and foot, heart rate (HR) and respiration.
We shall not use the entire dataset of seventeen drivers to study $[16,17]$ the stress degree, but to test the causal rate in the short and long term between predefined signals of these drivers. The general placement of sensors in automotive system is shown in Figure 1.

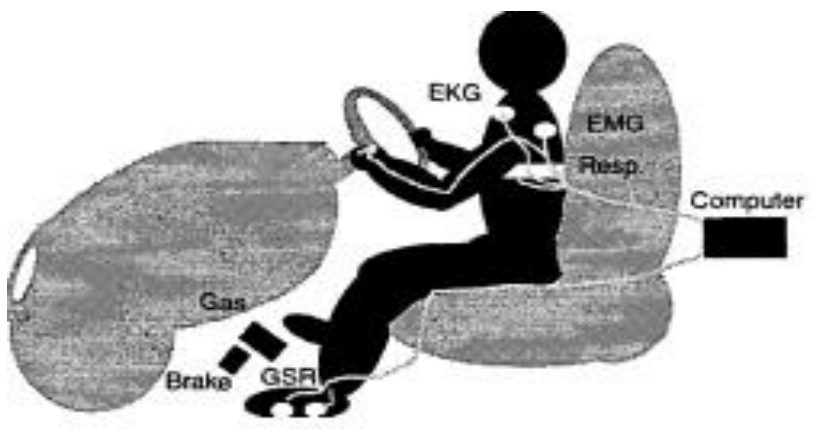

Fig. 1. Placement of sensors [29]

The final duration of the drive, with rest periods, varied from approximately $50 \mathrm{~min}$ to $1.5 \mathrm{~h}$ determined by traffic conditions. Drivers are questioned directly after each drive for filling out the subjective rating questionnaires. We placed the EMG on the trapezius muscle to indicate emotional stress [16] we measured the skin conductance in two places: in the middle and first finger of left hand with electrodes and on the left foot sole to measure the respiration we the expansion of chest cavity with an apropried sensor The EKG was sampled at 496 $\mathrm{Hz}$, the skin conductivity and respiration sensor were sampled at $31 \mathrm{~Hz}$, and the EMG was sampled at $15.5 \mathrm{~Hz}$ after first passing through a $0.5 \mathrm{~s}$ averaging filter. The signals were collected by an embedded computer in a modified car. The experimenter visually monitored the physiological signals as they were collected using a laptop PC running a remote display program. Figure 2 shows an example of the signals collected on a typical day's drive along with markings showing driving periods and events [29].

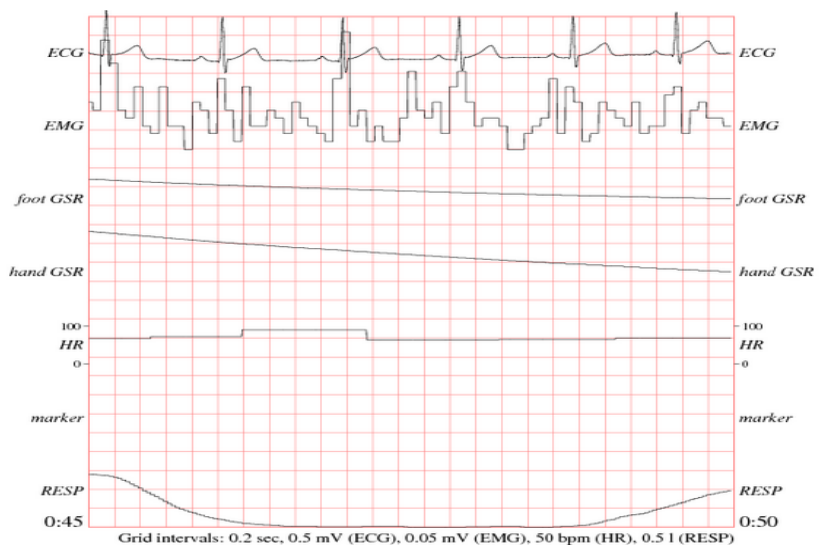

Fig. 2. Physiological data collected from Electrocardiogram (ECG), electromyogram (EMG), the respiration, heart rate, GSR foot and hand [16]

\section{Methodology}

Admittedly, the data models of panel have multiple advantages, but they do not seem sufficient any more to study all the phenomena, especially for our case resides in the study of the physiological signals. We must thus consider the last 
evolutions of the data of panel in term of multi-variety and non-stationariness in order to estimate our results correctly.

There exist a certain number of nonlinear models for data of panel, among which one can quote:

- Pooled Models.

- Fixed effect models.

\section{$\checkmark \quad$ Fixed effects estimation Models.}

$\checkmark \quad$ Existence of fixed effects Tests.

- Random effect models.

$\checkmark \quad$ Estimation of the models for random purposes.

$\checkmark$ Hausmann Tests.

- Probit and Logit.

- Tobit I and II.

- Panel Co-integration data.

In what follows, one will be interested in this last method in order to evaluate our contributions. We will restrict our study with the models of Co-integration, of data, of panel, with the estimators FM-OLS and DOLS like with the causality of Granger in the panel.

To carry out, the objective is set higher; we called on a methodological strategy pluri-methodology, an analysis by the method of the panel data, which allowed us to exploit dimensions individual and temporal. Our approach of analysis is in the following stages:

- Unit root tests.

- Panel Co-integration,

- FM-OLS and DOLS Estimators,

- Panel Granger Causality.

Before starting the empirical part, it is necessary to explain each approach.

\section{CO-INTEGRATION APPROACH}

There exist a certain number of tests for Co-integration as a panel. One can quote, Kao [37], Bai and Ng [38], Mackoskey and Kao [39], Westerlund [40, 41, 42, 43], Westerlund and Edgerton [44], Hank [45, 46], Gengenbach, Palm and Urbain [47], Gutierrez [48] as well as the tests of Pedroni [21, 22, 23].

In our study and taking into account the length of the important temporal dimension of the data, we chose to apply the approaches of Pedroni.

Pedroni [21, 22] proposed various tests aiming at apprehending the worthless assumption of absence of intraindividual Co-integration at the same time for homogeneous and heterogeneous panels. Breaking values appearing in this work being relating to the presence of only one regressor in the relations of Co-integration.

Pedroni proposes an extension if the relations of Cointegration understand more than two variables. It is starting from these last tests that one concentrates more, because our study takes into account several parameters at the same time for the model as a panel. The tests of Pedroni take into account heterogeneity by the means of parameters which can differ between the individuals. Thus, under the alternative assumption, there exists a relation of Co-integration for each individual, and the parameters of this relation of Co-integration are not necessarily the same ones for each individual of the panel. The taking into account of such a heterogeneity constitutes an undeniable advantage since in practice, it is rare that the vectors of Co-integration are identical of one individual to the other of the panel.

Pedroni suggests seven tests: four are based on dimension will intra individual and three on inter individual dimension. In these seven tests, the statistics are built on the basis as of residues of the relations of Co-integration and a certain number of estimators of parameters of nuisance. By way of an example, the parameter of nuisance corresponds to the conditional variance of long run individual of the residues. Let us note finally that the number of delays retained in the regressions of type ADF (Increased Dickey-Fuller) can vary between the individuals. In order to implement the various tests, Pedroni suggests a procedure in four stages:

- Stage 1: One estimates the relation of long run and one recovers the estimated residues.

- Stage 2: For each individual, one differentiates the series yit and one calculates the residues resulting from the following regression.

- Stage 3: The variance of long run is estimated.

- Stage 4: By using the estimated residues, one chooses the suitable regression.

\section{ESTIMATING THE LONG RUN RELATIONSHIP}

The assertion of the existence of a relation of Cointegration between the series must be followed, by the estimate of the relation of long run. Several techniques exist in this direction, Pedroni [21, 22] showed that the two methods FM-OLS (Fully Modified Ordinary Least Squares) and also the method of least squares dynamic DOLS (Dynamic Ordinary Least Squares), are the adequate methods with the evaluation of such a relation and who allow the convergent estimate of the parameters in a panel presenting a problem of endogeneity and non stationariness.

\section{A. FM-OLS}

This procedure makes it possible to take account of the problems of endogeneity of the second order of the regressors (generated by the correlation between the residue of Cointegration and the innovations of variables I (1) present in the relation of Co-integration) and of the properties of autocorrelation and heteroscedasticity of the residues. It is in addition advisable to note that this model of estimate of the relation of long run is used much if we have a significant number of data, such physiological signals in the medical field.

\section{B. DOLS}

The approach DOLS was initially suggested in the case of the time series, then adapted to the case of the data of panel. 
This technique consists in including values advanced and delayed in the relation of Co-integration, in order to eliminate the correlation between the explanatory variables and the term from error. The estimator DOLS has the same asymptotic distribution as estimator FM-OLS. This last show also a light superiority compared to the method DOLS. He is regarded as being the most robust technique in the estimate of the relations of panel Co-integration in the case of one a large number of data for the test. The representation of these two methods is illustrated in [49].

\section{PANEL GRANGER}

As we saw in the previous section, Co-integration in data of panel is a method which makes it possible to check the existence or the absence of the long-term relation between the variables. It does not specify the direction of causality. When a relation of Co-integration exists between the variables, it must be modelled in a model with correction of dynamic error. The principal goal of each study is to draw up causal links between the endogenous variable and the whole of the exogenous variables. The tests of causality of Granger are based on the following regressions:

$$
\begin{aligned}
(1-L)\left[\begin{array}{c}
X 1_{i t} \\
X 2_{i t} \\
X 3_{i t} \\
X 4_{i t} \\
\vdots i t \\
X n_{i t}
\end{array}\right]= & {\left[\begin{array}{c}
a_{i X 1} \\
a_{i X 2} \\
a_{i X 3} \\
a_{i X 4} \\
\vdots \\
a_{i X n}
\end{array}\right]+\sum_{i=1}^{P}(1-L)\left[\begin{array}{ll}
\vartheta_{11 i p} & \vartheta_{12 i p} \\
\vartheta_{21 i p} & \vartheta_{22 i p}
\end{array}\right]\left[\begin{array}{c}
X 1_{i t-p} \\
X 2_{i t-p} \\
X 3_{i t-p} \\
X 4_{i t-p} \\
\vdots \\
X n_{i t-p}
\end{array}\right] } \\
& +\left[\begin{array}{c}
\beta_{X 1_{i}} \\
\beta_{X 2_{i}} \\
\beta_{X 3_{i}} \\
\beta_{X 4_{i}} \\
\vdots \\
\beta_{X n_{i}}
\end{array}\right] Y_{t-1}+\left[\begin{array}{l}
\varepsilon_{1 t} \\
\varepsilon_{2 t}
\end{array}\right]
\end{aligned}
$$

Where $X 1, X 2, \ldots X n$ represent the explanatory variables, $p$ Lag (the length of delay), $(1-L)$ is the first operator of difference and $\mathrm{Y} \mathrm{t}-1$ signify the shifted term in correction of error coming from the relation of Co-integration. In order to illustrate all that, one proceeds to the empirical part relating to the theoretical aspect. One is interested first of all in the general information on the specification of the model used, concerning the various variables treated as well as the various panels of our study. Then, one follows in this part, the stages of the method of Co-integration in data of panel, while starting with the tests of the unit roots and while finishing by the test of causality of Granger in panel, all this for the three panels considered in our case.

The analysis of the biomedical signals nowadays is of an importance increased in the development of the medical therapeutic strategies. With the development of data processing and digital calculation, it becomes interesting to integrate an approach of assistance into the diagnosis in a computing process automatic. The choice of the mathematical models resulting from the signals for the characterization from such or such pathology becomes crucial then. We saw that in the previous chapter or decision making depends primarily on a certain number of parameters, difficult sometimes to extract.
In order to widen the detection of these anomalies, another vision is elaborate in this second contribution. It corresponds to the statistical analysis of observations regularly spaced in the time and in search of factors of causality between the physiological signals. More especially as these signals, generated by the displacement of an electric field in living tissue (EEG, EMG, ECG, etc), have multiple variations carrying relevant or harmful information to the extraction of medical information.

The criteria most used by the medical community are the measurement of time intervals (lasted of an event, separation of two events, delays) to characterize a temporal variation. This is why, we made a second contribution to develop a multivariate causal model between the cardiorespiratory myogalvanic signals. The end worked, after the distinction of the key factors which interest us to establish our goal, is the formulation of a robust statistical model which is a congruent representation of a stochastic process (unknown). In the world of the statistics/probabilities, there exist several models in this direction. Our approach in this article uses the models of causality within the meaning of Granger between the cardiorespiratory myogalvanic signals.

The causality concept represents the whole of the antecedents, which its intervention makes it possible to understand any phenomenon. In Mathematics, causality between two time series is generally studied in terms of the forecast improvement according to the characterization of Granger, or in aiming to impulsional analysis, according to the Sims principles.

In accordance with Granger sense, a series "causes" another series if the knowledge of the past of the first improves the forecast of the second. According to Sims, a series can be recognized like causal for another series, if the innovations of the first contribute to the variance of error of forecast of the second. Between these two principal modes of causality statistical characterization, the Granger approach is certainly that which had the most echoes among the mathematicians; it will thus be retained within the framework of this study. The base of the Granger definition is the dynamic relation between the variables. As indicated, it is stated in terms of improvement of the variable predictibility. For Granger and Sekkat, we cannot highlight causality without taking into account the "time" factor [19].

In our article, we begin the method of panel Co-integration in order to test the existence or absence of a long-term relation between our studied signals (cardiorespiratory electromyogalvanics signals) for the 17 drivers.

Its weak point is that it does not indicate the direction of this causality; hence, the necessity of tackling the so-called Panel Granger causal mathematical in order to model the directionality of the causality, the latter must be modeled in A dynamic error correction model of Engle and Granger [4].

Granger causality is one of the methods to model the idea of who causes the other, in other words, the idea that past effects help predict future effects. This concept was first traced by Wiener and implemented by Granger $[4,30,32]$ as a linear 
autoregressive vector model VAR, and later generalized by John Geweke [31].

The main purpose of each study is to establish causal links between the endogenous variable and all exogenous variables, Granger causality tests will be based on the following regression:

$(1-L)\left[\begin{array}{c}E C G_{i t} \\ E M G_{i t} \\ F O O T G S R_{i t} \\ H A N D G S R_{i t} \\ H R_{i t} \\ \text { respiration }_{i t}\end{array}\right]$

$$
\begin{aligned}
& =\left[\begin{array}{c}
a_{i E C G} \\
a_{i E M G} \\
a_{i F O O T G S R} \\
a_{i H A N D G S R} \\
a_{\text {iHR }} \\
a_{\text {iRESPIRATION }}
\end{array}\right] \\
& +\sum_{i=1}^{P}(1-L)\left[\begin{array}{ll}
\vartheta_{11 i p} & \vartheta_{12 i p} \\
\vartheta_{21 i p} & \vartheta_{22 i p}
\end{array}\right]\left[\begin{array}{c}
E C G_{i t-p} \\
E M G_{i t-p} \\
F O O T R_{i t-p} \\
H A N D G S R_{i t-p} \\
H R_{i t-p} \\
\text { respiration }_{i t-p}
\end{array}\right] \\
& +\left[\begin{array}{c}
\beta_{\text {ECG }_{i}} \\
\beta_{\text {EMG }_{i}} \\
\beta_{\text {FOOT GSR }_{i}} \\
\beta_{\text {HAND GSR }_{i}} \\
\beta_{\text {HR }_{i}} \\
\beta_{\text {RESPIRATION }_{i}}
\end{array}\right] E C T_{t-1}+\left[\begin{array}{l}
\varepsilon_{1 t} \\
\varepsilon_{2 t}
\end{array}\right]
\end{aligned}
$$

$p$ is the lag (the delay length), L-1 is the first difference operator and $E C T_{t-1}$ means the offset error correction term from the relationship of the Co-integration. An error correction model can distinguish between long-term and short-term relationship in the Granger causality. The short-term dynamics are captured by different coefficients of staggered terms.

The statistical significance of the coefficients of each explanatory variable is used to test Granger causality in the long term while the meaning of the coefficients of $E C T_{t-1}$ provides information on the short-term causality.

\section{EMPIRICAL RESULT}

Recent progress of mathematical modelling filled with enthusiasm the researchers, in particular in the panel data analysis. Methodology that we apply here is mainly based on the four fundamental parts of the panel data analysis, exposed previously. Initially, we applied unit roots tests to evaluate the series stationnarity.

The second phase consists to apply the Co-integration tests and to quantify later this long-term relation using FM-OLS and DOLS estimators. Finally, we applied the Granger causality tests to the whole of the studied panels.

Before beginning our results, we must clarify some details and signs:
$>$ Corresponds to the causal direction between our physiological signals.

$>$ this sign is a non-causal

$>$ A Value above the sign $\longrightarrow$, is the value of Fstatistic, which is considered a measure of the correlation between the variables studied.

$>$ A Value below the sign $\longrightarrow$, which is in brackets, corresponds to the value of the probability of causation.

$>$ Level: I (0).

$>$ First difference: I (1).

$>\{\mathrm{x}\}$ : Std Error (The standard error is the standard deviation of the sampling distribution of a statistic)

$>\quad *$ : Indicates statistical significance at $1 \%$.

$>\mathrm{x} \mathrm{E} \mathrm{y}:(x$ exponential $y)$.

$>$ [ ]: Long-term coefficient.

\section{A. Panel unit root tests}

In this section, we used the unit root tests on panel data (Levin Lin and Chu (LLC) [18], IM Pesaran and Shin (IPS) [19], Breitung (BRT), Maddala and Wu (MW) [20]). We will introduce you now; the results of LLC, IPS, BRT, MW and HADRI tests applied to the variables of our model. Our analysis of the results is mainly based on the Hadri test, which is known and recognized by its robustness, power and precision, it shows us without any ambiguity that the variables are non-stationary in level. Although the results of other tests used can be confusing given that reveals a level stationarity, this incompatibility is due to the statistical differences of the various tests used that can give distinct results. This divergence in the results and power of HADRI test requires us to test and verify the stationarity of our variable in first differences.

\section{B. Panel Co-integration}

After the checking of the non-stationarity for the all variables of the panel, we proceed to study the existence of one long-term relation between these variables, and this by applying the Pedroni Co-integration tests, which are based on the unit roots tests of estimated residues, trying now to test Cointegration for the signals. Pedroni [21, 22, 23] proposes two tests families, one realized in 1999 [21] resting on seven tests (four based on intra individual dimension and three on interindividual dimension) and another family of tests realized in 2004 [22], suggesting another four tests containing balanced statistics. The two categories of tests rest on the null hypothesis of absence of Co-integration. The co-integration of the variables depends on the value of the probability associated with each statistics (probability <0.01). Table.1 summarizes the results of the Pedroni Co-integration statistics. From the results of the Pedroni Co-integration tests, we can notice that among the whole of the statistical tests, all the probability values are less than $1 \%$ (they all are to 0.0000 ). Therefore, the whole of these tests shows the existence of a relation of Co-integration. 
TABLE I. PEDRONI CO-INTEGRATION FOR ECG

\begin{tabular}{|c|c|c|c|c|c|c|}
\hline \multirow[b]{2}{*}{ Pedroni Methods } & \multicolumn{3}{|l|}{ Within dimension } & \multicolumn{3}{|c|}{ Between dimension } \\
\hline & Test & Statistics & Prob & Test & Statistics & Prob \\
\hline \multirow{4}{*}{$\begin{array}{l}\text { Pedroni (1999) } \\
\text { [21] }\end{array}$} & Panel v-statistic & 27.52606 & $(0.000)^{*}$ & Group p-statistic & -43.57195 & $(0.0000)^{*}$ \\
\hline & Panel rho-statistic & -48.9354 & $(0.000)^{*}$ & $\begin{array}{l}\text { Group pp- } \\
\text { statistic }\end{array}$ & -24.29321 & $(0.0000)^{*}$ \\
\hline & Panel PP-statistic & -24.7533 & $(0.000)^{\star}$ & $\begin{array}{l}\text { Group ADF- } \\
\text { statistic }\end{array}$ & -68.54084 & $(0.0000)^{*}$ \\
\hline & Panel ADF-statistic & -46.7641 & $(0.000)^{*}$ & & & \\
\hline \multirow{4}{*}{$\begin{array}{l}\text { Pedroni (2004) } \\
\text { (Weighted statistic) } \\
{[22]}\end{array}$} & Panel v-statistic & 18.80569 & $(0.000)^{\star}$ & & & \\
\hline & Panel rho-statistic & -45.0907 & $(0.000)^{*}$ & & & \\
\hline & Panel PP-statistic & -23.7351 & $(0.000)^{*}$ & & & \\
\hline & Panel ADF-statistic & -46.4090 & $(0.000)^{*}$ & & & \\
\hline
\end{tabular}

\section{FM-OLS and DOLS estimations}

In the light of the projections carried out in non-stationary time series, the estimators of non-stationary panel data can still solve a certain number of problems, in particular on the level of the estimate and inference. To estimate Co-integrated variables systems, just like to carry out tests on the Co-integration vectors, it is necessary to use an effective estimate methods. FM-OLS and DOLS estimators proposed by Pedroni Kao and Chiang [24] and Mark and Sul [25].

The estimation of a Co-integration relation, if it exists, which connects the variables of the model in double index is established by the suitable method (FM-OLS and/or DOLS). The estimated parameters by one of these methods will be interpreted as being long run elasticities. It is important to emphasize that the DOLS method presents the disadvantage of reducing the number of freedom degrees of the studied variables, which leads to less reliable estimates. The estimation results are reported in the Table.
Table. 2 establishes long-term elasticity between variables of the model using FM-OLS and DOLS estimators. The modelling of within dimension enables us to take into account the heterogeneity of the coefficients in their temporal and/or individual dimension. The within estimator eliminates the individual specific effects.

By analyzing the estimated model by the FM-OLS regressor, The results for ECG-dependent suggests that an increase of $1 \%$ in EMG, FOOTGSR, HAND GSR, HR, and RESPIRATION implies a variation of ECG which take a value of $0.016248 \%, 0.007241 \%, 0.028366 \%, 0.000511 \%$ and $0.000110 \%$ respectively in the within dimension based on the method FMOLS. With same way, the result for ECG suggest that an increase of $1 \%$ in EMG, FOOT GSR, HAND GSR, HR and RESPIRATION implies a variation of ECG which take a value of $0.065684 \%, 0.014534 \%, 0.032800 \%, 0.000304 \%$, $0.005986 \%$ respectively in the between dimension based on the same method.

TABLE II. FM-OLSS AND DOLS FOR ECG

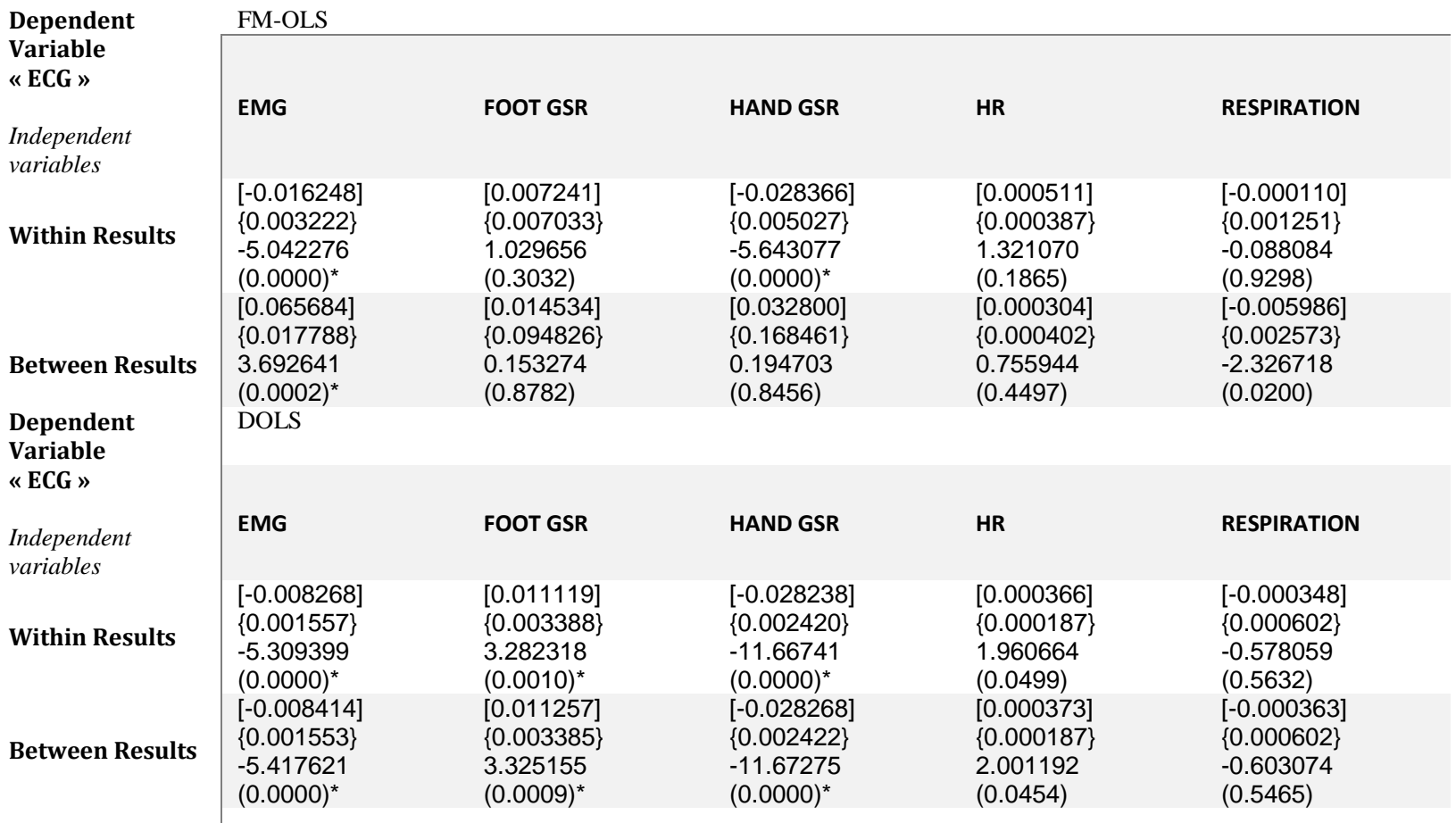




\section{Panel Granger causality}

The purpose of this part is to test the causal links between these variables using the Panel Granger causality test. A Granger causality analysis is carried out in order to determine if there is a power of potential foreseeability from one indicator to another. The results of the test for the all variables are summarized in table.3. It should be noted that the optimal delay (Lag) was established using the Akaike and Schwarz information criterion $[50,51]$.
The purpose of our study is to show the interactive relations between the whole of the signals, but that does not preclude under investigation of all possible relations. From the causality tests results presented in table.3, we can deduce the causal links direction which may appear between the variables in the threshold criticizes (probability of error) of $1 \%$. To be more explicit, if the probability is less than $1 \%$, we speak about a causal relation, in the opposite case, we speak about a no causal relation between variables.

TABLE III. PANEL GRANGER CAUSALITY

\begin{tabular}{|c|c|c|c|c|c|c|}
\hline Lag $=49$ & ECG & EMG & FOOT GSR & HAND GSR & HR & RESPIRATION \\
\hline ECG & & $\begin{array}{c}1.01343 \\
(0.4462)\end{array}$ & $\frac{3.76621}{(2 . E-13)^{*}}$ & $\underbrace{3.76640}_{(2 . E-13)^{*}}$ & $\begin{array}{c}0.93217 \\
(0.5853)\end{array}$ & $\overbrace{(5 . E-10)^{*}}^{3.17198}$ \\
\hline EMG & $\overbrace{(0.0001)^{*}}^{2.08195}$ & & $\begin{array}{c}0.23925 \\
(1.0000)\end{array}$ & $\begin{array}{c}0.22863 \\
(1.0000)\end{array}$ & $\begin{array}{c}0.07047 \\
(1.0000) \\
(1.000\end{array}$ & $\begin{array}{l}0.34500 \\
(0.9999)\end{array}$ \\
\hline FOOT GSR & $\overbrace{(2 . E-32)^{x}}^{6.73692}$ & $\begin{array}{c}0.30623 \\
(1.0000)\end{array}$ & & $\underbrace{446.983}_{(0.0000)^{*}}$ & $\begin{array}{c}0.08348 \\
(1.0000)\end{array}$ & $\underbrace{943.990}_{(0.0000)^{*}}$ \\
\hline HAND GSR & $\overbrace{(5 . E-33)^{*}}^{6.82513}$ & $\begin{array}{c}0.05842 \\
(1.0000)\end{array}$ & $\overbrace{(0.0000)^{*}}^{450.845}$ & & $\begin{array}{c}0.46971 \\
(0.9972)\end{array}$ & $\overbrace{(0.0000)^{*}}^{1002.34}$ \\
\hline HR & $\begin{array}{c}0.80450 \\
(0.7912)\end{array}$ & $\begin{array}{c}0.16269 \\
(1.0000)\end{array}$ & $\overbrace{(0.0005)^{*}}^{1.95451}$ & $\overbrace{(0.0014)^{*}}^{1.85638}$ & & $\underbrace{2.43276}_{(3 . E-06)^{*}}$ \\
\hline RESP & $\overbrace{(5 . E-25)^{x}}^{5.61495}$ & $\begin{array}{c}0.10165 \\
(1.0000)\end{array}$ & $\begin{array}{c}1.19212 \\
(0.1989)\end{array}$ & $\overbrace{(3 . E-95)^{*}}^{15.5611}$ & $\begin{array}{c}1.09573 \\
(0.3185)\end{array}$ & \\
\hline
\end{tabular}

Our study aims to illustrate the interactive relationships between all the variables EMG, GSR FOOT, HAND GSR, HR, RESPIRATION and the ECG signal, but that does not preclude the study of all possible relationships.

From the results of Granger Causality Test Panel presented in the table above, we can deduce the direction of causal relationships between variables can figure the critical threshold (error probability) of $1 \%$.

\section{DISCUSSION AND CONCLUSION}

In order to check the long-term convergence between our studied signals, we applied the Co-integration method; the results show that there is actually a convergence of these signals. In this article, we focus on the technical and nonmedical aspects in the fact that we belong to the telemedicine fields and we try to trace the short-and long-term causalities among drivers to take the necessary statistical information to prevent damage accidents. The knowledge and the quantitative understanding of these interactions are critical in monitoring people during driving, we wanted to study causality tests while driving in order to develop in next research an application telemedicine preventive, with perfect causal analysis of vital signs during driving.

These results and these statistical analyses will constitute at the same time a prediction base as well as a beginning of action towards new researches orientations treating the physiological interactions quantitatively. In this article, we concentrated much more on the technical sides and we leave the medical explanations to the health specialists to clarify this convergence/divergence, causality/no causality of the physiological signals.

The fact of studying these interactions, perhaps we can cure and prevent the sudden death which is quasi-unforeseeable and relentless, implying sudden demonstrations of some undesirable interactions in the human body, consequently it became a true syndrome in most unsuspected cases for the majority of the population (Nutritive, adult,...).

As prospects with these research tasks, we suggest the integration of several algorithms of the signal treatment, such as the causal processes, on a system embarked to appreciate these interdependences between physiological signals in the same application. The obtained results in this article pointed out the importance of improving the models existing in order to a better description for the various phenomena. However, in order to understand the mechanisms and to be able to prevent and treat more effectively these diseases, our studied models require to be improved by integration of the qualitative factors like the age, the sex, the diseases histories ... and that due to a certain number of mathematical models like Logit, Probit and Tobit.

We also plan to work out a model embarked to study panels more widened and with a very large number of patients gathered in blocks having the same symptoms, or the same age interval, or the same kind... 


\section{CONFLICT OF INTEREST}

The authors confirm that this article content have no conflict of interest.

\section{REFERENCES}

[1] E. Bekiaris, S. Nikolaou, "State of the Art on Driver Hypovigilance Monitoring and Warning Systems, AWAKE System for effective Assessment of driver vigilance and Warning According to traffic risk Estimation, " (IST-2000-28062). 2001 Nov; 20.

[2] Accidents de la route: 4540 morts et 69.582 blessés en Algérie en 2013 Available at: http://www.reflexiondz.net/ACCIDENTS-DE-LAROUTE-4540-morts-et-69-582-blesses-en-Algerie-en2013_a28409.html

[3] J. A. Horne, Reyner. L. A, "Counteracting driver sleepiness: effects of napping, caffeine and placebo. Psychophysiology," 33 (3): 306-309; 1996 May, doi: 10.1111/j.1469-8986.1996.tb00428.x.

[4] Granger. C. W. J, "Investigating causal relations by econometric and cross-spectralmethods, " Econometrica, 1969 Aug; 37 (3), 424-438.

[5] Yongmiao. Hong, Yanhui. Liu, Shouyang. Wang, "Granger causality in risk and detection of extreme risk spillover between financial markets, " Journal of Econometrics, 2009 june; 150 (2), 271-287.

[6] Pierre. Olivier. Amblard, Olivier. J. J. Michel, "On directed information theory and Granger causality graphs," J ComputNeurosci, 2011, 30,pp. 7-16.

[7] Xiang. Li, Kaiming. Li, Lei. Guo, Chulwoo. Lim, "Tianming Liu. Fiber-Centered Granger Causality Analysis. Medical Image Computing and Computer-Assisted Intervention - MICCAI 2011," Lecture Notes in Computer Science. 2011, 6892,pp. 251-259. doi: 10.1007/978-3-642-23629-7_31.

[8] Zhu. J, Chen. Y, Leonardson. A. S, Wang. K, Lamb, J. R, et al, "Characterizing dynamic changes in the human blood transcriptional network," PLoSComput. Biol. 2010, 6 (2) , e1000671. doi:10.1371/journal.pcbi.1000671.

[9] Pereda. E, R. Q. Quiroga, J. Bhattacharya, "Nonlinear multivariate analysis of neurophysiological signals," ProgNeurobiol. 2005,77 (12),pp. 1-37.

[10] Yifan. Zhao, Steve. A. Billings, Hua. Liang. Wei, Ptolemaios. G. Sarrigiannis, "A parametric method to measure time-varying linear and nonlinear causality with Applications to EEG data," IEEE, 2013, pp.1-7.

[11] M. Palu. S, A. Stefanovska. Phys. Rev. 2003; E 67, 055201R.

[12] Faes. L, L. Widesott, M. Del. Greco, R. Antolini, G. Nollo, "Causal cross-spectral analysis of heart rate and blood pressure variability for describing the impairment of the cardiovascular control in neurally mediated syncope," IEEE Trans Biomed Eng, 2006, 53 (1),pp. 65-73.

[13] Samir. Ghouali, Mohammed. Feham,Yassine. Zakarya. Ghouali, "The direction of information between Cardiorespiratory Hemodynamic signals: Test Analysis using Granger Causality," Journal of Mathematics, Statistics and Operations Research (JMSOR), 2(2), DOI: 10.5176/2251-3388_2.2.52. doi: 10.5176/2251-3388_2.2.52.

[14] Pereda. E, D. M. de. La. Cruz, L. De. Vera, J. J. Gonzalez, “Comparing generalized and phase synchronization in cardiovascular and cardiorespiratory signals," IEEE Trans Biomed Eng. 2005; 52 (4): 578583.

[15] Rosenblum, M. G, L. Cimponeriu, A. Bezerianos, A. Patzak, R. Mrowka, "Identification of coupling direction: application to cardiorespiratory interaction," Phys. 2002; Rev. E 65:041909.

[16] PHYSIOBANK ATM Available at: http://www.physionet.org/cgibin/atm/ATM.

[17] PhysioBank: Physiologic signal archives for biomedical research Available at: http://www.physiomet.org/physiobank.

[18] Levin. A, Lin. CF, Lin. ChuJ, "Unit root tests in panel data asymptotic and finite-sample properties," J Econometrics. 2002, 108 (1),pp. 1-24.

[19] Im. KS, Pesaran. MH, Shin. Y, "Testing for unit roots in heterogeneous panels," J of Econometrics, 2003, 115,pp. 53-74.
[20] Maddala. G. S, Wu. S, "A comparative study of unit root tests with panel data and a new simple test," Oxford Bulletin of Economics and Statistics Special Issue, 1999, 61, pp.631-652.

[21] Pedroni P, "Critical values for Co-integration tests in heterogeneous panels with multiple regressors. Oxford Bulletin of Economics and Statistics," 1999, 61, pp.653-678.

[22] Pedroni. P, "Panel Co-integration: asymptotic and finite sample properties of fooled time series tests with an application to the PPP hypothesis," Econometric Theory , 2004, 20,pp. 597-625.

[23] Pedroni. P, "Purchasing power parity tests in Co-integrated panels. The Review of Economics and Statistics," 2001 November, 83(4),pp. 727-731.

[24] Kao. C, Chiang. M. H, "On the estimation and inference of a Cointegrated regression in panel data," In: Baltagi, B.H. (Ed.), "Advances in Econometrics: Nonstationary Panels. Panel Co-integration and Dynamic Panels," 2000, 15, pp.179-222.

[25] Mark. N. C, Sul. D, "Co-integration vector estimation by panel DOLS and long-run money demand.Oxford Bulletin of Economics and Statistics," 2003 Dec, 65 (5),pp. 665-680.

[26] Nagal. D,Sharma. S,"Simultaneous 12-lead QRS detection by K-means clustering algorithm," Recent Advances and Innovations in Engineering (ICRAIE), 2014, pp.1 - 4, DOI:10.1109/ICRAIE.2014.6909244

[27] I. Elamvazuthi,N.H.X. Duy,Zulfiqar Ali,S.W. Su,M.K.A. Ahamed Khan,S. Parasuraman ,"Electromyography (EMG) based Classification of NeuromuscularDisorders using Multi-Layer Perceptron," Procedia Computer Science, 2015 IEEE International Symposium on Robotics and Intelligent Sensors (IEEE IRIS2015), Volume 76, 2015, Pages 223228.

[28] Nicola. Gerrett,Bernard. Redortier,Thomas. Voelcker, George. Havenith, "A comparison of galvanic skin conductance and skin wettedness as indicators of thermal discomfort during moderate and high metabolic rates," Journal of Thermal Biology, Volume 38, Issue 8, December 2013, Pages 530-538, http://dx.doi.org/10.1016/j.jtherbio.2013.09.003.

[29] Jennifer. A. Healey, Rosalind. W. Picard, "Detecting stress during realworld driving tasks using physiological sensors," IEEE Trans. Intelligent Transportations Systems, Vol. 6, No. 2, April 2005.

[30] Anil. K. Seth, Adam. B. Barrett, Lionel. Barnett,"Granger Causality Analysis in Neuroscience and Neuroimaging," The Journal of Neuroscience, February 25, 2015, 35(8):pp. 3293-3297.

[31] Geweke. J, "Measurement of linear dependence and feedback between multiple time series," J Am Statistical Assoc, 77, pp.304 -313,1982

[32] Barnett. L, Barrett. AB, Seth. AK, "Granger causality and transfer entropy are equivalent for Gaussian variables," Phys Rev Lett 103, 238701, 2009.

[33] Samir. Ghouali, Mohammed. Feham, Yassine. Zakarya. Ghouali, "Causal relationships between Cardiorespiratory Hemodynamics signals: Test Analysis using panel cointegration," The World Congress On Computer Applications and Information Systems (WCCAIS'2014), 2014 January 17-19, Tunisia, IEEE, pp 1-8. DOI: 10.1109/WCCAIS.2014.6916591.

[34] Samir. Ghouali, Mohammed. Feham, Yassine. Zakarya. Ghouali, "Revealing the Dynamic Correlation between Cardiac and Respiratory Hemodynamic Signals Using Time-Dependent Panel Co-Integration Analysis,". doi: 10.15662/ijareeie.2014.0311091, November 2014.

[35] Sami Khedhiri, "cours d'économétrie méthodes et applications", Lavoisier 2007 paris page 93.

[36] Hsiao, C, "Analysis of panel data", Cambridge University Press, 2003.

[37] Kao C, "Spurious Regression and Residual-Based Tests for Cointegrationin Panel Data", 1999, Journal of Econometrics, 90, pp. 1-44.

[38] Bai J, Ng S, "A panic Attack on Unit Roots and Cointegration", 2004, Econometrica, 72(4), pp. 1127-1178.

[39] McCoskey S. et Kao C, "A Residual-Based Test of the Null of Cointegrationin Panel Data”, 1998, Econometric Reviews, 17, pp. 57-84.

[40] Westerlund, J, "Testing for error correction in panel data", 2007, Oxford Bulletin of Economics and Statistics 69: 709-748.

[41] J. Westerlund, "New simple tests for panel Cointegration", Econometric Reviews, 24:297-316, 2005. 
[42] J. Westerlund, "Testing for panel Co-integrationwith a level break". Economics Letters, 91:27-33, 2006.

[43] J. Westerlund, "Panel Co-integrationtests of the Fisher Hypothesis", Journal of Applied Econometrics, 23:193-233, 2008.

[44] J. Westerlund and D. L. Edgerton, "Simple tests for Co-integrationin dependent panels with structural breaks", Lund University, Department of Economics, January 2007.

[45] C. Hanck, "Cross-sectional correlation robust tests for panel Cointegration", Universität Dortmund, SFB 475 Technical Report 44/06, Ruhr Graduate School in Economics, November 2006.

[46] C. Hanck,"A meta analytic approach to testing for panel Cointegration", Universität Dortmund, SFB 475 Technical Report 02/07, Ruhr Graduate School in Economics, January 2007.

[47] Christian Gengenbach, Franz C. Palm, Jean-Pierre Urbain, "CointegrationTesting in Panels with Common Factors", Oxford Bulletin of
Economics and Statistics, Volume 68, Issue s1, December 2006 Pages 683-719, DOI: 10.1111/j.1468-0084.2006.00452.x.

[48] L. Gutierrez, "Simple tests for Co-integrationin panels with structural breaks", Applied Economics Letters, 2008.

[49] Roberto Basile , Mauro Costantini , Sergio Destefanis, "Unit root and cointegration tests for cross-sectionally correlated panels. Estimating regional production functions," ISAE Working Paper No. 53. Available at $\quad$ SSRN: $\quad$ https://ssrn.com/abstract=936324 http://dx.doi.org/10.2139/ssrn.936324

[50] Renaud Lacelot, Matthieu Lesnoff, "Sélection de modèles avec l'AIC et critères d'information dérivés," Version 3, Novembre 2005.

[51] Henry de-Graft Acquah, "Comparison of Akaike information criterion (AIC) and Bayesian information criterion (BIC) in selection of an asymmetric price relationship," Journal of Development and Agricultural Economics Vol. 2(1) pp. 001-006, January 2010, Available online at http://www.academicjournals.org/JDAE ISSN 2006- 9774. 